\title{
Effects of Exploratory and Heuristic Multi-methods on System-oriented Curricula Based on Clinical Scenarios
}

\author{
Chunting $\mathrm{Lu}^{1, \#}$, Simin Huang ${ }^{2, \#}$, Zejian $\mathrm{Li}^{3, \#}$, Lie Feng ${ }^{2} \&$ Jing Yang ${ }^{2}$ \\ ${ }^{1}$ The Science \& Education Office, the First Affiliated Hospital, Jinan University, Guangzhou, China \\ ${ }^{2}$ Department of Internal Medicine, First Clinical Medicine College of Jinan University, Guangzhou, China \\ ${ }^{3}$ Medical Centre of Stomatology, the First Affiliated Hospital of Jinan University, Guangzhou, China \\ \#: These authors have contributed equally to the work. \\ Correspondence: Jing Yang, Department of Internal Medicine, First Clinical Medicine College of Jinan University, \\ Guangzhou, 510630, China.
}

Received: October 26, 2017

Accepted: November 9, 2017

Online Published: November 14, 2017

doi:10.5430/ijhe.v6n6p55

URL: https://doi.org/10.5430/ijhe.v6n6p55

\begin{abstract}
A system-oriented curriculum in which basic medical courses and clinical subjects are integrated and reformed is a new trend for medical education in China. In order to train and enhance the comprehensive clinical competency, thirty-two excellent medical students who formed a observation group from grade 2010 in Jinan University were selected to participated in this study. Three different exploratory and heuristic pedagogies, which employed the flexible application of case-based learning (CBL), problem-based learning (PBL), and team-based learning (TBL), were explored and implemented in cardiovascular, respiratory, and digestive teaching sections. The effects of these exploratory and heuristic pedagogical approaches based on the flexible application of the CBL, PBL, and TBL methods with clinical scenarios in system-oriented curricula are satisfactory. Accordingly, these teaching methods and experiences had been summarized and promoted to mainland and non-mainland medical students from grade 2014 in Jinan University. Teaching and learning effects are also satisfactory. In addition, different characteristics in conducting these courses between two types of university students are further compared and analyzed for the improvement of all system-oriented curricula in the future.
\end{abstract}

Keywords: Clinical scenarios, System-oriented curricula, Case-based learning (CBL), Problem-based learning (PBL), Team-based learning (TBL)

\section{Introduction}

The goal of the national program to foster excellent has provided good opportunities for medical students and has promoted innovations in clinical education in the five-year clinical medicine major (Jin \& Zhang, 2011). This study explores and evaluates the effects of a new teaching mode that integrates basic medical science with clinical curricula through the study of human systems. This research focuses on a flexible application of multiple methods, including case-based learning (CBL), problem-based learning (PBL) (i.e., Chen, Lai, Lu, Tsai, Chiang, Huang, \& Yu, 2008; Jones, McArdle, \& O'Neill, 2002; Oda, Koizumi, 2008; Ali et al., 2015; ), and team-based learning (TBL), and examines their respective advantages by closely combining them with clinical cases (see, e.g., VanLehn, 1999; Atkinson, Derry, Renkl \& Wortham, 2000; Paas \& Van Gog, 2006; Stark, Kopp \& Fischer, 2011; Pan, Cheng, Zhou, Li \& Yang, 2017).

During the teaching and learning process, real, appropriate clinical cases have been merged into the system-oriented curriculum through the integration of basic medical subjects with clinical knowledge. In the past decade, practice-based learning and improvement (PBLI) and systems-based practice (SBP) have proven to be the most challenging competencies to teach and assess since these requirements went into effect (Carey \& Colby, 2013). Therefore, systems-oriented courses can be conducted in the fifth- or sixth-year classes of the clinical medicine major after medical students have finished the basic medical sciences in the third year. The CBL, PBL and TBL approaches are used flexibly and emphasize the importance of understanding clinical situations with basic medical theory (Yu \& Wang, 2011). This understanding is most important and extremely helpful for closely combining fundamental theory with clinical skills applications, which can increase students' clinical thinking abilities and skills in using basic medical knowledge. Exploratory and heuristic approaches can stimulate students' interest and 
encourage them to constructively explore clinical problems. It is important to positively cultivate an interdisciplinary system in the 5-year undergraduate clinical medicine major that is guided by actual clinical issues to overcome the traditional education disadvantages that separate basic medical science from clinical practice. The skills that students acquire in clinical practice are consistent with the skills that are identified as essential to professional practice. In addition, a common big picture concept was iterated throughout the themes and demonstrated that case-based learning prepares students for the multifaceted problems that they are likely to encounter in professional practice (Harman, et al., 2015.). Accordingly, the goal of this study is to train medical students' active learning, information processing, teamwork, problem-solving, doctor-patient communication skills, decision-making capacity, etc.

Furthermore, these teaching methods can be summarized and promoted to more medical students in the university. Jinan University, in Guangzhou, China, recruits Chinese students from the mainland, overseas Chinese students and foreign students. In this study, the students from mainland China are referred to as mainland students, whereas other students are referred to as non-mainland students and may come from Hong Kong, Macau, Taiwan, Malaysia, Myanmar, the Philippines, Thailand, the United States, Canada, Peru, etc. Based on these characteristics, it is necessary to compare and study the differences between the teaching features for mainland and non-mainland students, including motivation, performances in the classroom and hospital, clinical skills training, and the evaluation forms of undergraduates to update educational ideas, adapt students' aptitude and improve their learning efficiency. Accordingly, teaching and learning strategies can be explored and researched to play an important role in guiding medical education.

\section{Method}

\subsection{Participants and Setting}

Thirty-two excellent students in grade 2010 whose total scores ranked them at the top of their class were selected to establish a specific class as the observation group. They were in their third year of study and had completed theoretical and practical courses in basic medical science, diagnostics, medical images, etc. In this year of study, the cardiovascular, respiratory, and digestive sections of the system-oriented curriculum were established and taught using exploratory and heuristic approaches including CBL, PBL, and TBL. Finally, the effects of these teaching and learning modes were analyzed. After analysis, these programs were expanded to the students in grade 2014, including mainland students (five-year higher education) and non-mainland students (six-year higher education).

Seventeen mainland students in grade 2014 participated in cardiovascular and respiratory system-oriented courses, while 13 students participated in a urinary system-oriented course. A total of 47 mainland students in this class successively selected system-oriented courses.

Twenty-six non-mainland students in grade 2014 participated in a respiratory system-oriented course, while 36 students participated in a system-oriented course, and 25 students participated in a digestive system-oriented course. A total of 87 non-mainland students in this class successively selected system-oriented courses.

\subsection{Procedures}

The arrangements of the curriculum for grades 2010 and 2014 are illustrated in Table 1. Each section on common cardiovascular, respiratory, and digestive diseases was designed and begun based on real clinical cases by using bedside teaching and group discussions and presentations. Simultaneously, basic and clinical knowledge was introduced and systemically integrated through interdisciplinary cooperation between basic knowledge and clinical teachers. A total of 45 teaching hours were arranged for each system.

Table 1. Arrangement for each system-oriented course

\begin{tabular}{ll}
\hline Items & Teaching Hours (h) \\
\hline Basic medical science related & 20 \\
Clinical lecture related & 9 \\
Bedside practice & 7 \\
Integrated and comprehensive study including CBL, PBL and TBL & 9 \\
\hline
\end{tabular}

2.2.1 Research on System-oriented Curricula in the Specific Class of Grade 2010

2.2.1.1 Cardiovascular system-oriented integrated curriculum

In the first step, the students were arranged in the ward to observe a patient with acute chest pain. The teachers supervised them in collecting clinical information by taking the patient's history, performing a physical examination 
and demonstrating familiarity with laboratory and instrumental investigations according to clinical thinking principles. Then, the students came back to the classroom, where the teachers explained and reviewed basic medical knowledge including anatomy, histology and embryology, pathophysiology, pathology, ECG analysis, etc. In this part of the class, CBL was predominant, as the real clinical situation may stimulate the students' learning interests.

In the second step, the students were organized into a case discussion using the PBL approach for the first time, with the arrangement as follows.

1) Groups were established, and the teachers and students were introduced to one another.

2) The content, purpose and requirements of the case discussion were introduced and guided by the teachers.

3) Each student in the group reported and shared his or her thinking related to diagnostic and therapeutic information, learning resources, and the problems that needed to be discussed in the classroom.

4) Case-based and problem-based discussions were held, and numerous questions were discussed by the groups.

5) The teachers and students collected the questions that were generated during the discussions and refined them according to the teaching syllabus to arrange future learning activities.

In the third step, the teachers addressed some of the students' questions and explained key points relative to the differential diagnoses of acute chest pain and the diagnoses and management of coronary artery disease. Then, the teachers guided the students to watch a percutaneous transluminal coronary angioplasty (PTCA) that was performed on the patient who had been received on the first day. Therefore, the analysis and thinking in the group discussions could be confirmed or excluded after the students observed the operation. This method may greatly increase student interest and impressions regarding this real case.

In the fourth step, the students were active in stating their own views in the second PBL discussion class because they were quite familiar with the case by this time. Finally, the teachers summarized the main problems and the essentials of the diagnosis and treatment of this real case, and they also offered comments on the students' performances and the evaluation scores of individuals and groups. Therefore, a combination of PBL and TBL approaches was applied at this time.

\subsubsection{Respiratory system-oriented integrated curriculum}

In the first step, the teacher provided to the students clinical information and materials that included the teaching requirements of a respiratory case. Then, the students were organized and divided into several groups. The first PBL discussion class in this system then began. During group discussions, the teachers gradually gave more clinical information according to the students' comprehension and encouraged the students to take responsibility for their own learning. Afterward, the teacher assigned tasks to further consult the literature and explore answers.

In the second step, respiratory system-oriented subjects and knowledge, including anatomy, pathology and physiology, microbiology, immunology, and pharmacology, were reviewed by the teachers of basic medical science, while the key points of respiratory symptoms, signs, laboratory tests, and images were explained by the clinical teachers.

In the third step, the students were brought to the respiratory ward to learn and practice basic clinical skills such as history taking and physical examinations. After they accumulated this clinical information, the students returned to the classroom for the second PBL discussion. The teachers encouraged the students to consider and analyze the diagnostic clues and to create a future management plan for pleural effusion.

In the fourth step, the teachers offered a general overview of common respiratory diseases. The students were divided into several groups for another discussion. Finally, the teachers summarized the students' performances and provided the key points of this case.

\subsubsection{Digestive system-oriented integrated curriculum}

The first step of this system was to provide clinical materials to the students for critical thinking and analysis. Then, the team of basic and clinical teachers gave lectures to help the students to review the relevant knowledge of the anatomy, physiology, biochemistry, medical images, and diagnostic essentials of common digestive disorders.

The students voluntarily discussed in groups and sought more study materials. Following this discussion, the second step was to arrange for the students to go to the ward and the endoscopy room to recognize and learn the clinical features of and the diagnostic and therapeutic technology for digestive diseases. The third step was a PBL discussion that was introduced by a real case. Finally, the students reported the results of the group discussion, and the teachers summarized the students' performances and provided the key points of this case. 


\subsubsection{Assessment methods for grade 2010}

After the course, an open-book paper exam was given regarding the case analysis that related to these three system disorders. During the exam, the students could review materials online. If the students passed, the appropriate certificate was awarded. In addition, each student was asked to complete the feedback form, "Evaluation Form for the System-oriented Curricula Effects of Exploratory and Heuristic Methods".

\subsubsection{System-oriented course arrangement expanded to grade 2014}

After we explored and summarized the teaching experience with the system-oriented curricula in grade 2010, we expanded this experience to grade 2014, which comprised mainland and non-mainland medical students in their first year of study at Jinan University. The arrangement and teaching hours were the same as those for grade 2010. The selected teaching methods were similar to the methods that had been practiced and that had shown the best and most popular results in the cardiovascular system-oriented course in the prior class. Accordingly, the students were first brought to the ward to observe a patient, and the teachers supervised them in collecting clinical information by taking the patient's history, performing a physical examination and demonstrating familiarity with laboratory and instrumental investigations according to clinical thinking principles. Then, the students returned to the classroom, where the teachers explained and reviewed basic knowledge, including anatomy, histology and embryology, pathophysiology, pathology, ECG analysis, etc. Afterward, group discussions were conducted by using a combination of the CBL, PBL, and TBL methods, and then diagnosis and treatment were also discussed. As the class progressed, the students could review the related literature and find relevant problem-solving methods. Finally, the teachers offered a conclusion and evaluation of the students' performances.

2.2.2.1 For the mainland class, the cardiovascular, respiratory, and urinary system curricula were conducted in order. After the course, an open-book paper exam was given concerning the case analysis that related to these three system disorders. During the exam, the students could review materials online.

2.2.2.2 For the non-mainland class, the respiratory, cardiovascular, and urinary system curricula were conducted in order. After the course, an open-book paper exam was given on the case analysis that related to these three system disorders. During the exam, the students could review materials online.

2.2.2.3 The mean scores of the final examinations for grade 2014 were compared, including the scores of both the mainland and non-mainland students for different system courses.

2.2.2.4 The differences in the mean scores of the system-oriented course exams were compared between the mainland and non-mainland classes.

\subsection{Statistical Analysis}

Statistical analyses were performed using SPSS 17.0 (SPSS Inc., Chicago, Illinois). The data conformed to a normal distribution and were expressed as Mean \pm SD; the data were analyzed by independent sample t-tests. Two-tailed p-values of $<0.05$ were considered to be statistically significant.

\section{Results}

\subsection{Basic Information in Grade 2010}

All students in the class of grade 2010 passed the theory-based exam of case analysis, and the appropriate certificates were awarded. In total, 32 feedback forms were distributed, and the recovery rate was $100 \%$. The 20 -question questionnaire was designed to determine the students' responses to and suggestions for this teaching and learning mode (as shown in Table 2). 
Table 2. Effectsof System-oriented Curricula by Exploratory and Heuristic Methods in grade 2010 (n=32)

Questions
1. Do you understand CBL, PBL, and TBL?
2. Are you interested in CBL, PBL, and TBL?
3. Do you think that exploratory and heuristic methods
are helpful to individual learning?

4. Do you think that exploratory and heuristic methods can promote clinical thinking ability?

5. Do you think that exploratory and heuristic methods can promoteactive learning?

6. How long is the most suitable duration for independent study in each system?

7. Do you think that exploratory and heuristic methods can promote clinical decision-making ability?

8. Do you think that exploratory and heuristic methods can improve team-work and cooperation

9. Are you clear the objectives and requirements of the courses?

10. Do you think that it is necessary to discuss the syllabus by both teachers and students together?

11. Do you think what are the roles of teachers played in CBL, PBL, and TBL?

12. Are you satisfactory with the mode of integration between basic medical science and clinical knowledge?

13. Are you satisfactory with the arrangements among lecture, bedside practice, and case discussion?

14. Do you think whether the problems generated during the course or discussion can be solved well or not afterward?

15. How many times considered the most suitable to PBL discussions in each disease unit?

16. Do you think whether learning resources and approaches enough or not?

17. Do you think it is necessary forexploratory and heuristic methods merging into clinical skills training?

18. How is exploratory and heuristicteaching effect?

19. Which one is the best mode among these three system-oriented curricula?

20. What is the total impression of all arrangements?

Items of Assessment and Percentages (\%)

Well understood

(1/3.13)

Greatly interested

(22/68.75)

Greatly helpful

(25/78.13)

Greatly promote

(28/87.50)

Greatly promote

(26/81.13)

2 hours

(9/28.13)

Greatly promote

$(15 / 78.13)$

Greatly improve

(17/53.13)

Clear

(10/31.25)

Very necessary

(19/59.38)

Dominant

$(3 / 9.38)$

Very satisfactory

$(9 / 28.13)$

Very satisfactory

$(7 / 21.88)$

Very well

$(13 / 40.63)$

Twice

(21/65.63)

Enough

(16/50.00)

Very necessary

(28/87.50)

Good

(6/18.75)

Cardiovascular

(23/71.88)

Good

(26/80.00)
Moderately understood

(29/90.63)

Moderately interested

(10/31.25)

Moderately helpful

(6/18.75)

Moderately promote

(4/12.50)

Moderately promote

(5/15.63)

5hours

(17/53.13)

Moderately promote

(6/18.75)

Moderately improve

(15/46.88)

Moderately Clear

(20/62.50)

Necessary

(8/25.00)

Helpful

(17/84.38)

Satisfactory, yet

further improved needed

(21/65.63)

Satisfactory

(20/62.50)

Fair

(17/53.13)

Three times

(11/34.38)

Fair

(15/46.88)

Necessary

(2/6.25)

Fair, yet

further improved needed

(22/68.75)

Respiratory

(8/25.00)

Fair

(6/20.00)
Poorly understood

(2/6.25)

Poorly interested

(0/0)

Poorly helpful

(1/3.13)

Poorly promote

$(0 / 0)$

Poorly promote

(1/3.13)

Others

(6/18.75)

Poorly promote

(1/3.13)

Poorly improve

(0/0)

Not clear

(2/6.25)

Not necessary

(5/15.63)

None

(2/6.25)

Not satisfactory

$(2 / 6.25)$

Not satisfactory

(5/15.63)

Not solved

(2/6.25)

Others

$(0 / 0)$

Not enough

(1/3.13)

Not necessary

(2/6.25)

Not satisfactory

(4/12.50)

Digestive

(1/3.13)

$\mathrm{Bad}$

$(0 / 0)$ 


\subsection{The Mean Scores of the Final Examinations for Mainland Students in Grade 2014}

The comparison of the mean scores of the final examinations for the mainland students in grade 2014 during each system-oriented course of study is illustrated in Table 3 and Figure 1.

Table 3. Comparison of the mean scores of final examination of mainland students in grade 2014 for different system course study.

\begin{tabular}{lllll}
\hline Cardiovascular system $(n)$ & Respiratory system $(n)$ & Urinary system $(n)$ & F & $P$ \\
\hline $74.15 \pm 9.70(17)$ & $77.88 \pm 7.65(17)$ & $84.92 \pm 6.10(13)$ & 6.85 & 0.004 \\
\hline
\end{tabular}
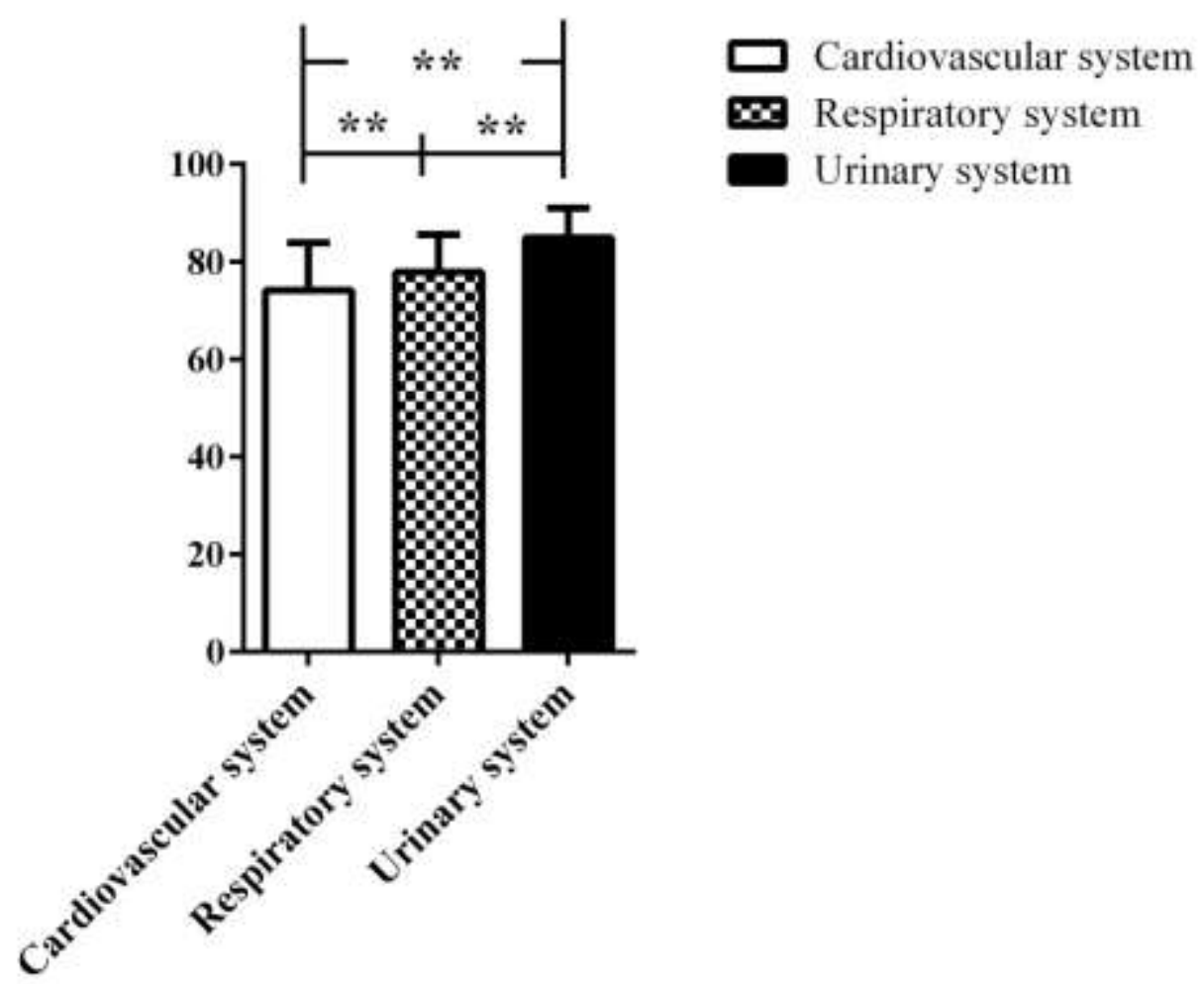

Figure 1. Comparison of the mean scores of final examination of mainland students in grade 2014 for different system course study. $* * p<0.01$ between two system course study.

The collected data were analyzed by using repeated measurement analysis of variance. There were statistically significant differences $(P<0.05)$ among the cardiovascular, respiratory, and urinary system-oriented courses according to the order in the mainland class.

\subsection{The Mean Scores of the Final Examinations for Non-mainland Students in Grade 2014}

The comparison of the mean scores of the final examinations for the non-mainland students in grade 2014 during each system-oriented course of study is illustrated in Table 4 and Figure 2.

The collected data were analyzed using repeated measurement analysis of variance. There were statistically significant differences $(P<0.05)$ among the respiratory, cardiovascular, and digestive system-oriented courses according to the order in the non-mainland class.

Table 4. Comparison of the mean scores of final examination of non-mainland students in grade 2014 for different system course study.

\begin{tabular}{lllll}
\hline Respiratory system $(n)$ & Cardiovascular system $(n)$ & Digestive system $(n)$ & F & $P$ \\
\hline $65.81 \pm 14.55(26)$ & $73.33 \pm 11.39(36)$ & $85.50 \pm 6.58(25)$ & 21.58 & 0.000 \\
\hline
\end{tabular}




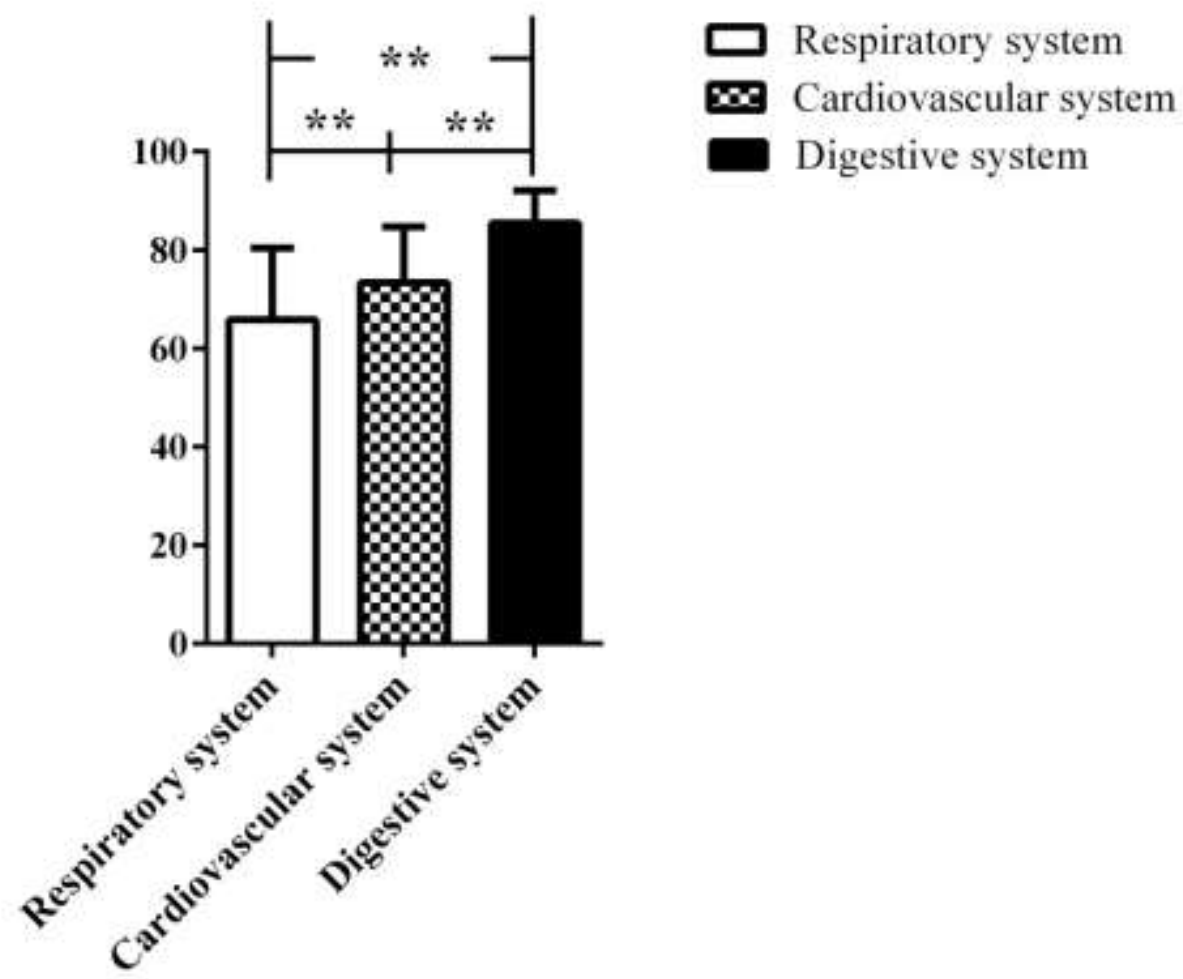

Figure 2. Comparison of the mean scores of final examination of non-mainland students in grade 2014 for different system course study. $* * p<0.01$ between two system course study.

3.4 The Differences in the Three System-oriented Courses of Study between the Mainland and Non-mainland Classes in Grade 2014

The comparison of the differences in the mean scores during all three system-oriented courses of study between the mainland and non-mainland classes is illustrated in Table 5 and Figure 3.

Table 5. Comparison of the mean scores during all three system-oriented courses study between mainland class and non-mainland class.

\begin{tabular}{lllll}
\hline & Mainland class & Non-mainland class & $t$ & $P$ \\
\hline Total scores(47/87) & $78.48 \pm 9.03$ & $74.58 \pm 13.60$ & 1.98 & 0.049 \\
\hline
\end{tabular}




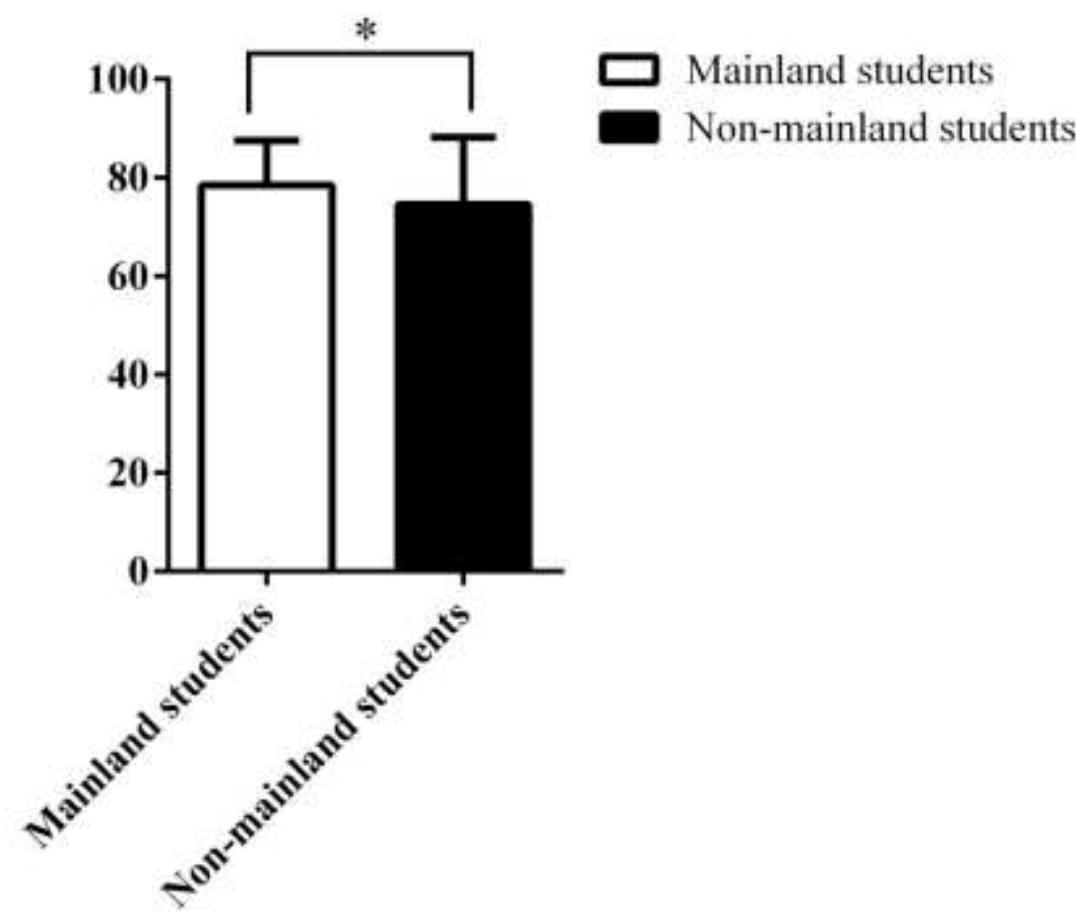

Figure 3. Comparison of the mean scores during all three system-oriented courses study between mainland class and non-mainland class. ${ }^{*} p<0.05$ between two classes.

\section{Discussion}

\subsection{The Application of the CBL, PBL, TBL Teaching Methods in Grade 2010}

In the class of grade 2010, the flexible application of the CBL, PBL, and TBL teaching methods based on organs and systems was explored and practiced. These methods were combined with real clinical scenarios, and cardiovascular, respiratory, and digestive system-oriented curricula were established to increase the interaction between the teachers and students and to encourage the students to consider and solve real clinical questions, including questions regarding the diagnosis and treatment of common diseases, using active learning, teamwork and cooperation. Moreover, in the teaching process, more practical opportunities were provided to train the students to improve their clinical comprehension abilities. This research focused on studying the characteristics, advantages and timing of different exploratory and heuristic methods that are adapted to actual clinical scenarios. For example, CBL was used to motivate students' enthusiasm for learning in the early stage of the system-oriented courses. As students advanced in their mastery of clinical practices, the PBL and TBL methods were applied and encouraged the students to consider the clinical problems and to actively participate in the discussions. The students' comments and suggestions regarding the exploratory and heuristic methods were collected and analyzed for further improvement.

4.1.1 The results from the "Evaluation Form for the System-oriented Curricula Effects of Exploratory and Heuristic Methods in Grade 2010" showed that the system-oriented curricula that involved the flexible application of the CBL, PBL, and TBL methods were popular with the students and helped them to consider clinical problems and to discover problem-solving methods, which enhanced their clinical thinking and clinical skills. Most students (81.13\%) agreed that the exploratory and heuristic methods promoted independent study. In addition, the new teaching mode was considered to greatly improve clinical thinking ability $(87.5 \%)$, clinical decision-making capacity $(78.13 \%)$, and teamwork and cooperation (53.13). Moreover, $84.38 \%$ of the students thought that the teachers played a guiding, not dominant, role in the PBL discussion. Overall, $87.5 \%$ of the students believed that exploratory and heuristic methods are necessary to merge clinical skills with operational training. Meanwhile, $80 \%$ of the students' impressions of the entire arrangement were positive. These results all indicate that the results of the teaching and learning approaches that relate to reforming the system-oriented curricula by using exploratory and heuristic methods are satisfactory.

4.1.2 In the teaching process, the cardiovascular system-based integrated curriculum was the most popular, and $71.88 \%$ of the responses were positive. The reasons for this preference may relate to the setting of the lecture, practice, and case discussion in the ward and the fact that teaching began in a real clinical scene using CBL, which allowed the students to learn and perform basic clinical skills simultaneously. In addition, the teachers of the basic medical 
course gave specific lectures according to clinical situations, which allowed basic medical theory and clinical skills to be reviewed and integrated. All of these elements can increase students' positive impressions of the course. Afterward, the first PBL and TBL discussion was arranged to promote the students' active learning and discovery of problem-solving methods. This discussion helped them to experience clinical thinking and to establish a preliminary clinical diagnosis and treatment steps. During this exploratory and heuristic teaching and learning, the students also had the opportunity to observe the diagnostic and therapeutic technology and understand their therapeutic effectiveness in a real case. Therefore, this mode optimized the practical clinical process that began with basic training, clinical practice, the integration of basic knowledge with clinical knowledge, and enhanced clinical ability, and the students returned to the clinical situation, which confirmed the ideas that were discussed by individuals and in group discussions. The teachers further analyzed and summarized the key points of diagnosis and the case management plans in the subsequent class. Moreover, $65.63 \%$ of the students believed that having two PBL discussions on a topic is appropriate.

4.1.3 Half of the students considered the learning resources and approaches sufficient, whereas $46.88 \%$ considered them fair. The internet has made reviewing information and study materials convenient and easy for students. The key problem was to improve their ability to screen, integrate and analyze the information that was obtained.

\subsubsection{Teaching Problems in Need of Improvement}

Most of the students (90.63\%) only showed a basic understanding of the CBL, PBL, and TBL methods, and 62.50\% of the students had a moderately clear understanding of the teaching objectives and requirements. Therefore, it was very necessary $(59.38 \%)$ or necessary $(25 \%)$ for the teachers and students to discuss the syllabus of the system-oriented curricula together to better understand exploratory and heuristic methods. Overall, $65.63 \%$ of the students believed that the integration methods of basic medical knowledge with clinical knowledge must be improved, and $68.75 \%$ believed that the evaluation forms for the exploratory and heuristic teaching effects should be also improved. Accordingly, preparatory work should be conducted, including lesson planning and discussions by teachers of basic medical knowledge and clinical skills, etc. It is necessary to design and simplify the key points that were taught in the lectures and increase clinical skills practice to consolidate the students' clinical basis and avoid repeated content. In addition, the final evaluation should focus more on which formative assessments increased the proportion of the performances in teamwork and cooperation to encourage students to actively participate in the discussions.

After the above results and problems were analyzed, system-oriented courses were developed for both the mainland and non-mainland classes in grade 2014. The teaching and learning methods that were similar to the most popular program in the cardiovascular system curriculum were applied. Real clinical cases and scenarios were introduced at the beginning, and the integration of pre-clinical knowledge with basic clinical knowledge followed. Gradually, problem-solving methods were explored through both self-study and guidance from the teacher, and eventually, diagnostic, therapeutic and decision-making abilities were improved. A comparison of the pedagogical approaches among the three system-oriented courses produced statistically significant results. These results showed that teaching and learning efficiency improved through comprehensive step-by-step training, which progressed from the cardiovascular to the respiratory to the urinary system in the mainland class but progressed from the respiratory to the cardiovascular to the digestive system in the non-mainland class. Exploratory and heuristic methods can be better mastered and applied. We will also continue to explore and observe the impacts of these modes on the students' along with the following clinical study in grade 2014.

A comparison of the mean scores of the final examinations between the mainland and non-mainland classes in grade 2014 indicated higher and statistically significant $(P<0.05)$ scores for the mainland students. In the usual teaching process, the mainland students demonstrated stronger individual learning ability, a better basis for understanding theoretical and clinical knowledge, and more active motivation. Most of them wanted to be clinical doctors in the future. These factors likely encouraged the mainland students to study hard in their clinical courses. In contrast, the non-mainland students at Jinan University had more personality diversity. They were active in thinking and liked having case studies more than pure theoretical study. Furthermore, some non-mainland students were not as clear regarding their expectations for the future, and some were concerned about their future career. For this type of student, study enthusiasm may be poor. Accordingly, it is important to find the common interests of these two types of students, enhance their self-study capacity, and promote their thinking concerning clinical problems. It is also necessary to encourage them to participate in group discussions and cultivate their teamwork abilities. The entire program should focus on training students in professionalism regardless of what occupation they choose in the future. 


\subsection{Teaching Innovation and Expansions of the Experience are Helpful}

In the actual teaching process, it was found that it is difficult for a single, simple approach to obtain positive teaching effects. Therefore, it is necessary to recognize the advantages and disadvantages of different approaches. A flexible application of the CBL, PBL, and TBL methods may be helpful to improve the clinical comprehension ability of medical students. These modes of instruction can motivate students' active learning, teamwork and cooperation. According to the results of our study, good teaching experiences and results have been obtained in specific classes of the five-year clinical medicine major. In the future, the program can be designed and expanded to the entire clinical medicine college to allow more students to participate in this type of course. Then, group discussions and training can be based on real clinical cases with the CBL, PBL and TBL methods to emphasize the importance of basic medical science in understanding clinical conditions. These approaches are particularly suitable to Chinese medical education. Interdisciplinary curricula can be applied throughout each stage of medical education, such as early clinical experience courses, integrated system-oriented basic and clinical courses, clinical case analysis courses, etc. During each course, the CBL, PBL, and TBL approaches can be flexibly applied.

In the early stage of undergraduate education, teamwork and cooperation should be emphasized by a TBL approach after guiding students in the hospital. In this period, it is important to allow students to understand clinical situations and express their own thinking concerning medical ethics and professionalism, the doctor-patient relationship, etc. Team-based learning improves student perception of some but not all areas of communication, teamwork, and professionalism (Elmore, Skelley \& Woolley, 2014). It is also essential to stimulate students' interest and enthusiasm to learn the basics of medical science. As basic and clinical knowledge is increasingly mastered in the middle stage of undergraduate education, PBL approaches can be employed that focus on cultivating students' ability to find and solve the problems that are generated by clinical conditions. Therefore, students can learn and master medical science knowledge by merging practical problems with it. When students enter the late study period, CBL combined with group discussions can be adopted to further enhance their clinical comprehension and analysis abilities.

In each course, teams of outstanding teachers who consist of interdisciplinary faculty can be established. Each system-oriented curriculum can be established based on clinical problems, common symptoms, such as fever, abdominal pain, headaches, palpitations, etc., or real-life cases. All teachers should pay more attention to updated teaching methods and focus on refining or designing appropriate problems that pertain to real clinical scenarios. All of these elements can motivate students' interest and abilities in problem-solving, innovation, the application of theory to practice, their active participation in discussions, the careful consideration of clinical issues, clinical skills practice, etc. The aim is to train students to become outstanding physicians in the future.

\section{Conclusion}

The effects of exploratory and heuristic pedagogical approaches that use the flexible application of the CBL, PBL, and TBL methods to clinical scenarios in system-oriented curricula are satisfactory. Different characteristics between the mainland and non-mainland students had been found and analyzed for the future improvement of teaching and learning.

\section{Limitations and Future Directions}

The main purpose of this study focuses on the exploration and development of system-oriented and integrated curricula; however, not all the original teaching plans for undergraduates can be changed. We respect students' own optional courses. Therefore, researchers' analyses can only emphasize the mean scores of each class. In this case, it is difficult to compare the progression of the test scores for each student in successive courses because only several students selected all three system-oriented courses. To perfect medical education, it is necessary to develop better teaching plans and to continue to optimize the curricula of clinical medicine. We will attempt to arrange the system-oriented courses more scientifically and reasonably in the future.

\section{Acknowledgments}

We thank the large number of academic staff and medical students who contributed to the research and the usual teaching in first clinical medicine college and medical college of Jinan University. The authors alone are responsible for the content and writings of this article. The authors appreciated the help of Dr. Yanghai Zhou from the first affiliated hospital of Jinan University and Professor Renbin Qi from the department of pathophysiology, school of basic medicine, Jinan University and Sanqiang Pan from the Department of Clinical Medicine, Medical College, Jinan University. 


\section{Funding Information:}

This work was supported by Teaching Research Programs of Higher Medical Education of Ministry of Education in 2016 (grant number: Edu-CR-2016-4-2, Edu-CR-2016-4-3 and Edu-CR-2016-6-2), Education Teaching Reform Project of Guangdong Province (grant number: 85514022), Graduate Education Innovation Project of Guangdong Province (grant number: 82616302), Education and Teaching Reform Project of Jinan University (grant number: 51071289, JG2015116 and JG2016028) and Fundamental Research for the Central Universities (grant number: 216174100)

\section{Compliance with Ethical Standards:}

Declaration of Interest: The authors have no conflicts of interest to report.

Ethics and consent: The research was conducted in accordance with the Helsinki Declaration of 1975, as revised in 2008. The medical student participants were informed in written and oral remarks. During the whole process, the patients' privacy must be considered and protected, and no invasive and harmful procedures were performed on them. In addition, the data of the study were accessible to the authors.

\section{References}

Ali IA, Mohamed TA, Al Kabba AF, Alotaibi SS, Al Shehri AM, Abdulghani HM \& Badri M .(2015). Problem-based learning in undergraduate medical education in Saudi Arabia: Time has come to reflect on the experience. Medical Teacher, 1(37), 61-66. https://doi.org/10.3109/0142159X.2015.1006608

Atkinson RK, Derry SJ, Renkl A \& Wortham D. (2000). Learning from examples: instructional principles from the worked examples research. Review of Educational Research, 70(2), 181-214. https://doi.org/10.3102/00346543070002181

Carey WA \& Colby CE. (2013). Educating fellows in practice-based learning and improvement and systems-based practice: The value of quality improvement in clinical practice. Journal of Critical Care, 28(1), 277. https://doi.org/10.1016/j.jcrc.2012.07.003

Chen, C.S., Lai, C.S., Lu, P.Y., Tsai, J.C., Chiang, H.C., Huang, I.T., \& Yu, H.S. (2008). Performance anxiety at English PBL groups among Taiwanese medical students: a preliminary study. The Kaohsiung Journal of Medical Sciences, 24(3 Suppl), S54-58. https://doi.org/10.1016/S1607-551X(08)70095-0

Elmore L, Skelley J \& Woolley T. (2014). Impact of adapted team-based learning methods on student self-assessment of professionalism, teamwork, and skills in a self-care course. Currents in Pharmacy Teaching and Learning, 6(4), 488-493. https://doi.org/10.1016/j.cptl.2014.04.002

Harman T, Bertrand B, Greer A, Pettus A, Jennings J, Wall-Bassett E \& Babatunde OT. (2015). Case-Based Learning Facilitates Critical Thinking in Undergraduate Nutrition Education: Students Describe the Big Picture. Journal of the Academy of Nutrition \& Dietetics, 115(3), 378-388. https://doi.org/10.1016/j.jand.2014.09.003

Jin J \& Zhang GW. (2011). Application of PBL+LBL teaching methods to eight-year medical students in gynecology and obstetrics clinical teaching (in Chinese). Basic Medical Education, 27(2), 613-618.

Jones, A., McArdle, P.J., \& O'Neill, P.A. (2002). Perceptions of how well graduates are prepared for the role of pre-registration house officer: a comparison of outcomes from a traditional and an integrated PBL curriculum. Medical Education, 36(1), 16-25. https://doi.org/10.1046/j.1365-2923.2002.01105.x

Oda, Y., \& Koizumi, S. (2008). Status of medical education reform at Saga Medical School 5 years after introducing PBL. The Kaohsiung Journal of Medical Sciences, 24(3 Suppl), S46-53. https://doi.org/10.1016/S1607-551X(08)70094-9

Paas F \& Van Gog T. (2006). Optimising worked example instruction: different ways to increase germane cognitive load. Learning and Instruction, 6(2), 87-91. https://doi.org/10.1016/j.learninstruc.2006.02.004

Stark R, Kopp V \& Fischer MR. (2011). Case-based learning with worked examples in complex domains: Two experimental studies in undergraduate medical education. Learning and Instruction, 21(1), 22-33. https://doi.org/10.1016/j.learninstruc.2009.10.001

Pan SQ, Cheng X, Zhou YH, Li K \& Yang XS. (2017). Seeking the optimal time for integrated curriculum in Jinan University School of Medicine. International Journal of Higher Education, 6(1), 25-33. https://doi.org/10.5430/ijhe.v6n1p25

VanLehn K. (1999). Rule-learning events in the acquisition of a complex skill: an evaluation of Cascade. Journal of the Learning Sciences, 8(1), 71-125. https://doi.org/10.1207/s15327809jls0801_3

Yu SW \& Wang YS. (2011). Integrated application of LBL, PBL and TBL teaching methods in medical education(in Chinese). China Higher Medical Education, 5, 100-102. 